\title{
Ecoepidemiología de la Leishmaniasis cutánea no ulcerada en Honduras
}

\author{
Wilfredo Sosa-Ochoa, ${ }^{1,4}$, Xochitl Morales Cortedano², Silvia Argüello², \\ Concepción Zuniga ${ }^{3}$, Jessica Henríquez ${ }^{3}$, Rosa Mejía ${ }^{3}$, Angel Mejía ${ }^{4}$, \\ Gabriela Araujo ${ }^{4}$, Carmen Sandoval ${ }^{4}$, Doris Quan ${ }^{4}$
}

\section{RESUMEN}

La leishmaniasis es una enfermedad infecciosa causada por un protozoo del género Leishmania. En Honduras es una importante causa de morbilidad. El propósito del estudio fue caracterizar la eco epidemiología de la leishmaniasis cutánea no ulcerada (LCNU) en Honduras. Se seleccionaron 4 municipios endémicos a LCNU (Amapala, San Francisco de Coray, Reitoca y Alubarén).

Para realizar la captura de Lutzomyia se usaron trampas miniatura tipo CDC, los individuos capturados fueron identificados según los criterios de Young \& Duncan. Se realizaron búsquedas de pacientes en conjunto con personal de la Secretaría de Salud de Honduras para realizar la caracterización de las especies de Leishmania usando PCR. Se determinó la ingesta alimenticia de las hembras ingurgitadas utilizando la amplificación de ITS de los principales animales domésticos y humanos. Se colectaron 156 muestras de pacientes con sospecha clínica de LCNU 130 (83.33\%), resultaron positivas a Leishmania spp., siendo Leishmania infantum/chagasilaúnica especie circulante en lazona.

Por medio de la utilización de criterios de Young \& Duncan, se logró identificar un total de 5,951 Lutzomyia, de la cuales 3,543 (60 \%) son machos y 2,404 (40\%) son hembras con o sin ingesta de sangre. De esta población de insectos capturados, se identificaron diez especies de Lutzomyia, siendo Lu. longipalpis, Lu. evansi y Lu. cruciata las de mayor predominio. Los cuatro municipios presentaron una baja diversidad de especies de Lutzomyia $\left(D_{M g}=1.04\right)$. Se analizaron 450 hembras ingurgitadas de las especies: Lu. longipalpis, Lu. cruciata, Lu. evansi, Lu. chiapanensis y Lu. sanguinaria. Las principales fuentes alimenticias encontradas

\footnotetext{
1 Universidad Nacional Autónoma de Honduras. Facultad de Ciencias, Escuela de Microbiología, Grupo de Investigación de Parasitología. Correo electrónico: will.sosa.ochoa@gmail.com; wilfredo.sosa@unah.edu.hn

2 Universidad Nacional de Costa Rica. Maestría en Enfermedades Tropicales.

${ }^{3}$ Secretaría de Salud de Honduras.

${ }^{4}$ Universidad Nacional Autónoma de Honduras. Facultad de Ciencias, Escuela de Microbiología, Departamento de Investigación de Parasitología. Correo electrónico: will.sosa.ochoa@gmail.com; wilfredo.sosa@unah.edu.hn
} 
fueron: Gallus gallus, Sus scrofa, Canis familiaris. Se encontró una tasa de infección del $0.27 \%$, encontrándose a Lu. longipalpis infectada con Leishmania infantum/ chagasi. Con este estudio se logró caracterizar la eco epidemiología de la LCNU en Honduras, confirmando a $L$. chagasi/infantum como el principal agente etiológico y a $L$ u. longiplapis su principal vector.

Palabras clave: Lutzomyia longipalpis, Lutzomyia evansi, Leishmania infantum/chagasi, fuentes alimenticias

\section{ABSTRACT}

Leishmaniasis is an infectious disease caused by the Leishmania protozoon. In Honduras, it's an important cause of morbidity. This study's objective was to characterize the Eco-epidemiology of Non Ulcerated Cutaneous Leishmaniasis (NUCL) in Honduras. Four NUCL endemic counties were selected (Amapala, San Francisco de Coray, Reitoca y Alubaren).

In order to capture the Lutzomyia, CDC traps were used according the Young \& Duncan criteria for the taxonomic identification. The patients were found in conjunction with the Honduran Ministry of Health to execute the molecular characterization of the Leishmania species using PCR. Also, through the ITS amplification, we determined that those female sandflies, fed mainly from domestic animals and human blood. 156 samples from clinical suspicion for NUCL from which $130(83.33 \%)$ were positive for Leishmania spp, and Leishmania chagas/infantumi was the only characterized specie.

Using the Young \& Duncan criteria, 5,951 sandflies were identified, 3,543 (60\%) male and 2,404 (40\%) females. Among the captured insects, 10 sandflies species were identified and the most predominant were $L u$. longipalpis, $L u$. evansi and $L u$. cruciata. The four counties presented low sandflies specie diversity $\left(D_{M g}=1.04\right) .450$ engorged by these species were analyzed: Lu. longipalpis, Lu. cruciata, Lu. evansi, Lu. chiapanensis and Lu. sanguinaria. The main food sources found were: Gallus gallus, Sus scrofa and Canis familiaris. The infection rate found was of $0.27 \%, L u$. longipalpis infected with Leishmania infantum/chagasi. With this study, the ecoepidemiology of NUCL was characterized, confirming the L. chagasi/infantum as the main etiologic agent, Lu. longipalpis as its main vector, and Canis familiaris as the insects' main food source.

Keywords: Lutzomyia longipalpis, Lutzomyia evansi, Leishmania infantum/chagasi, Blood Meal. 


\section{INTRODUCCIÓN}

En Honduras existen cuatro formas clínicas de las leishmaniasis: cutánea ulcerada, muco cutánea, visceral y cutánea no ulcerada. Las formas de leishmaniasis cutánea difusa y recidivante son excepcionales. Se considera a las diferentes formas clínicas como un grupo de enfermedades que se distribuyen en forma endémica en varias zonas geográficas, afectando poblaciones rurales que penetran a zonas boscosas y húmedas (leishmaniasis cutánea ulcerada y muco cutánea) o bien zonas semidesérticas y secas (leishmaniasis visceral y cutánea no ulcerada) para establecerse en viviendas precarias en donde el vector se encuentra en su hábitat natural. Existe un subregistro de los casos, ya que gran número de los afectados viven en zonas alejadas y de difícil acceso a los servicios de salud.

En nuestro país, la leishmaniasis se encuentra distribuida en 12 departamentos (Programa Nacional de Leishmaniasis, 2007), siendo la cutánea no ulcerada la forma más común de la enfermedad, en la zona sur del país, cuyo principal agente etiológico es L.infantum/chagasi.

La primera descripción de esta nueva forma clínica de leishmaniasis en Honduras ocurrió en el municipio de Amapala, Isla del Tigre, al observarse (Ponce, 1991) dichas lesiones cutáneas en niños, familiares y vecinos de pacientes con leishmaniasis visceral y que no respondían a tratamientos contra otras enfermedades, principalmente la lepra. A la mayoría de los pacientes con este tipo de leishmaniasis les llaman leprosos y es un fenómeno de estigmatización y discriminación social por parte de la comunidad donde residen.

Además, uno de los principales riesgos de padecer este tipo de manifestación clínica de la leishmaniasis, es el proceso hacia la visceralización en niños menores de cinco años con problemas nutricionales, ya que el Laboratorio de Referencia de Leishmaniasis de la Secretaría de Salud de Honduras no cuenta con una prueba basada en la detección de ácidos nucleicos para parásitos del género Leishmania, por lo que este estudio servirá para adquirir y desarrollar nuevas herramientas de diagnóstico y vigilancia epidemiológica y transferirlas al Laboratorio Nacional de Leishmaniasis para poder tomas las medidas necesarias en la detección oportuna y vigilancia de la leishmaniasis cutánea no ulcerada.

Existen más de 900 especies de insectos flebotomíneos (Lutzomyia y otros géneros relacionados) que sirven como vectores a diferentes agentes etiológicos. Para el caso de la leishmaniasis cutánea no ulcerada, se asume que Lutzomyia longipalpis es el principal vector en Honduras, pero lo antiguo del último reporte exige una 
actualización a la luz de posibles cambios en el medioambiente y de la utilización de mejores técnicas de estudio. La identificación de las especies de insectos se hace tradicionalmente con claves entomológicas haciendo énfasis en características fenotípicas (Young y Duncan,1994) bajo microscopía de luz.

Debido a los cambios climáticos existentes y a las migraciones dentro del mismo país, es necesario determinar la ecoepidemiología de las especies de Leishmania y Lutzomyia circulantes en las zonas endémicas.

\section{MATERIALES Y MÉTODOS}

\section{Pacientes}

Las muestras clínicas fueron obtenidas de pacientes con lesiones características de la leishmaniasis cutánea no ulcerada recolectadas por personal del Laboratorio Nacional de Vigilancia y del Programa Nacional de Prevención y Control de la Enfermedad de Chagas y Leishmaniasis de la Secretaría de Salud de Honduras en los municipios de Amapala y San Francisco de Coray, en el departamento de Valle; Reitoca y Alubarén, en el departamento de Francisco Morazán, durante los meses de febrero a junio de 2012.

\section{Muestras clínicas}

Se realizó una búsqueda activa en los cuatro municipios para encontrar pacientes que presentaran lesiones compatibles con leishmaniasis. Para tales efectos, se definió a la lesión compatible con leishmaniasis cutánea no ulcerada, como una pápula, placa o nódulo asintomática, no ulcerativa, eritematosa o del color de la piel, con o sin presencia de un halo hipopigmentado.

La búsqueda activa la realizó el personal del Laboratorio Nacional de Vigilancia y de la Secretaría de Salud, que son los encargados de entregar el diagnóstico a los pacientes y proporcionar el medicamento a los pacientes que resulten positivos. Además, se les solicitó una lámina con 3 frotis sin ninguna identificación del paciente, para hacer el diagnóstico molecular de especies de género Leishmania por medio de la PCR.

Colección de Lutzomyias e identificación taxonómica

Especímenes de Lutzomyia fueron capturadas en cuatro municipios endémicos a

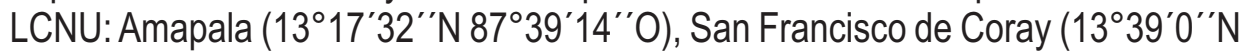


$\left.87^{\circ} 31^{\prime} 0^{\prime \prime} \mathrm{O}\right)$, Reitoca $\left(13^{\circ} 49^{\prime} 0^{\prime \prime} \mathrm{N} 87^{\circ} 28^{\prime} 0^{\prime \prime} \mathrm{O}\right.$ ) y Alubarén ( $13^{\circ} 47^{\prime} 45^{\prime \prime} \mathrm{N} 87^{\circ} 28^{\prime} 08^{\prime \prime}$ O). Se colocaron dos trampas miniatura tipo CDC en cada casa muestreada (una en el peridomicilio y otra en intradomicilio), que utilizan como atrayente luminoso luz blanca de 6 voltios sin atrayente químico; a una altura entre $30 \mathrm{~cm}$ y 1 metro sobre el suelo, para la captura de los insectos.

Las trampas totalmente armadas se colocaron a partir de las 18:00 horas hasta las 6:00 horas del día siguiente. La separación de la captura total de insectos recolectados por las trampas tipo CDC, se llevó a cabo mediante la utilización de estereomicroscopio con una magnificación de 100X, observando estructuras externas de las Lutzomyias, tales como tamaño, color, patas largas, posición de las alas, para su posterior identificación taxonómica.

Se colocaron en frascos con etanol al $70 \%$ por localidad, sexo y ubicación de la trampa (intra o peridomiciliar), se tuvo el cuidado de colocar las hembras grávidas e ingurgitadas por separado. Los machos fueron identificados por los genitales, ya que se pueden visualizar las estructuras reproductivas externas y para las hembras se utilizó la identificación de la espermateca y el cibario, según los criterios de clasificación de Young y Duncan (1994).

Posteriormente a la identificación, se formaron grupos de 5 hembras según especie, localidad y sitio de colecta, para determinar infecciones naturales y determinación de preferencias alimenticias de las especies identificadas. Los grupos de hembras formados fueron guardados en tubos de $1.5 \mathrm{ml}$ y guardados a $-20^{\circ} \mathrm{C}$ para extraer AND.

\section{Métodos moleculares}

El ADN de los frotis cutáneos y de los grupos de Lutzomyia fueron extraídos según protocolos del fabricante (DNeasy Blood y Tissue Kit, Hilden Germany).

Detección especies de Leishmania por medios de PCR: el AND estraído directamente de los frotis cutáneos y de los grupos de Lutzomyia fueron amplificados usando los cebadores 13A (5'-GTGGGGGAGGGGCGTTCT-3') Y 13b (5'-ATTTTACACCAACCCCCAGTT-3') amplificando un segmento de 120 pb del minicírculo del kinetoplasto de especies de Leishmania (Rodgers y otros, 1990).

Para determinar especies de Leishmaniam subgénero Viannia, se usaron los cebadores MP1L (5'-TACTCCCCGACATGCCTCTG-3') y Mp3 (5'-GAACGGGGTTTCTGTATGC-3') amplificando un segmento de 70 pb del minicírculo del kineto- 
plasto de especies de Leishmania, subgénero Viannia (Lopez y otros, 1993).

Para amplificar ADN de Leishmania (L.) infantum/chagasi se utilizaron los cebadores RV1 (5'-CTTTTCTGGTCCCGCGGGTAGG-3') y RV2 (5'-CACCTGGCCTATTTTACACCA-3') amplificando un segmento de $145 \mathrm{pb}$ del minicírculo del kinetoplasto de Leishmania (L.) infantum/chagasi (Lachaud y otros, 2002). La reacción de amplificación fue desarrollada con un volumen final de $25 \mu \mathrm{L}$ conteniendo Master Mix (PROMEGA), $0.6 \mu \mathrm{mol} / \mathrm{L}$ de cada cebador y $5 \mu \mathrm{L}$ de AND.

Caracterización de especies de Leishmania por medio de PCR-RFLP: el ADN extraído de las muestras de frotis cutáneo fue evaluado para identificar la especie de Leishmania involucrada en la infección en los seres humanos. Para este propósito se utilizó un ensayo de PCR-RFLP.

La amplificación inicial fue realizada usando los cebadores LSUC (5'CAAACTGGGGGTTGGTGTAA-3') y LSUL (5'-TTTTGAACGGGGTTTCTG3') que amplifican una región fluctuante de $600-800 \mathrm{pb}$ del minicírculo KDNA, dependiendo de la especie de Leishmania (Bhattacharyya y otros, 1996). La reacción de amplificación fue desarrollada con un volumen final de $50 \mu \mathrm{L}$ conteniendo $25 \mu \mathrm{L}$ de Master Mix (PROMEGA), $0.6 \mu \mathrm{mol} / \mathrm{L}$ de cada cebador y $10 \mu \mathrm{L}$ de AND.

Para determinar las especies de Leishmania se tomaron $15 \mu \mathrm{L}$ del producto de PCR y se realizó una digestión con $10 \mathrm{U}$ de enzima Haelll y $10 \mathrm{U}$ de Rsal (Promega, Madison, WI). La digestión fue realizada en un baño maría a $37^{\circ} \mathrm{C}$ durante 2 horas, con un volumen final de $20 \mu \mathrm{L}$. La visualización de los patrones fue realizada por medio de un fotodocumentador BioDoc-It ${ }^{\circledR}$ Imaging System (UVP, Upland, CA). Las amplificaciones fueron desarrolladas en tubos de $0.2 \mathrm{ml}$ con un termociclador modelo 2400 (Perkin-Elmer, Norwalk, CT).

Determinación de preferencias alimenticias de especies de Lutzomyia: el ADN extraído de las hembras ingurgitadas fue amplificado en una mezcla de $25 \mu \mathrm{L}$ compuesta por $12.5 \mu \mathrm{L}$ de Master Mix (PROMEGA), $0.6 \mu \mathrm{mol} / \mathrm{L}$ de cada cebador y 5 $\mu \mathrm{L}$ de ADN. A cada grupo de Lutzomyia se evaluaron los siguientes cebadores: aves, ratón, rata, perro, cerdo, humano; siguiendo el protocolo establecido por Walker y colaboradores $(2002,2003)$ (ver cuadro 1). 
Cuadro 1. Secuencias de cebadores y tamaño del fragmento esperado en la amplificación por especie de mamífero analizado

\begin{tabular}{|c|c|c|c|}
\hline $\begin{array}{l}\text { Orga- } \\
\text { nismo }\end{array}$ & Cebador I & Cebador II & $\begin{array}{l}\text { Tamaño } \\
\text { fragmen- } \\
\text { tado (pb) }\end{array}$ \\
\hline Cerdo & 5'GACTAGGAACCATGAGGTTGCG 3' & 5'AGCCTACACCACAGCCACAG 3' & 134 \\
\hline Aves & 5'CTGGGTTGAAAAGGACCACAGT 3' & 5'GTGACGCACTGAACAGGTTG 3' & 169 \\
\hline Perro & 5'AGGGCGCGATCCTGGAGAC 3' & 5'AGACACAGGCAGAGGGAGAA 3' & 83 \\
\hline Gato & 5'AGTCGGTTAAGCGTCTGACTTT 3' & 5'CTCCAGGCTCTGAGCTGTCA 3' & 98 \\
\hline Rata & 5'CAAGACGGATGATCAAAATGTG 3' & 5'ATTGGGTGGCTGTATATGTATGG 3' & 161 \\
\hline Ratón & ATGGCTCAGTGGGTAAAGG 3’' & 5'GTGGAGGTCAGAGGACAAACTT 3' & 118 \\
\hline Humano & 5'GAGATCGAGACCACGGTGAAA 3' & 5'TTTGAGACGGAGTCTCGTT 3' & 200 \\
\hline
\end{tabular}

\section{Caracterización macroambiental}

A partir de los datos históricos de temperatura media anual, precipitación anual y evapotranspiración potencial registrados para los cuatro municipios (SERNA, 2010), se determinó la zona de vida según el sistema Holdrige. También se ubicó espacialmente a los municipios con el software ArcView 3.3 y se caracterizó macroabientalmente con la información disponible del Atlas Digital de Honduras.

La caracterización macroambiental se hizo por medio de capas temáticas con la técnica asignación de datos (Spatial Join), utilizando la extensión geoprocesadora de ArcView (Xtools.aux) (ESRI, 2002). Las capas incluidas en la caracterización macroambiental fueron: geología, tipos de suelo y zonas de vida. Todas las capas se proyectaron en GWS 84, en una escala de 1:500 000 según los datos disponibles en elAtlas.

\section{Análisis estadístico}

Se definió la riqueza específica $(S)$ como el número total de especies presentes en cada localidad. Se determinó la abundancia proporcional de especies de Lutzomyia mediante la fórmula: $A_{i}=(n / N) \times 100$; donde $A_{i}$ es la abundancia proporcional de la especie $i$ con respecto a la abundancia total, $n$ es el número de individuos de la especie i y Nel número total de individuos (Moreno, 2001).

La diversidad de especies de Lutzomyia en cada localidad se calculó por medio del índice de Margalef (DMg) (Moreno, 2001). Su fórmula es la siguiente: 


$$
\operatorname{Dmg} \frac{S-1}{\operatorname{In} N}
$$

Donde:

S: número de especies de la comunidad.

$N$ : número total de individuos de la comunidad.

Para comparar la similitud en cuanto a composición de especies entre las cuatro localidades estudiadas, se estableció el coeficiente de Jaccard $(I J)$, el cual se expresa de la siguiente manera: $l_{j}=c / a+b-c$, donde $I_{j}$ es el índice de semejanza de Jaccard, a es el número de especies presentes en el sitio $\mathrm{A}, b$ es el número de especies presentes en el sitio $B$ y c el número de especies comunes para los sitios $A$ y $\mathrm{B}$. El rango de valores para este índice va de 0 cuando no hay especies compartidas entre los dos sitios o comunidades, hasta 1 cuando ambos sitios tienen la misma composición de especies (Moreno, 2001).

Para calcular la tasa de infección natural en flebótomos se consideró la siguiente razón:

$T I \frac{\text { número de poole de cada especies positivos para género Leishmania }}{\text { número total de pooles de esa especie }}$

\section{RESULTADOS}

\section{Pacientes}

Se colectaron 156 muestras de pacientes con sospecha clínica de leishmaniasis cutánea no ulcerada, distribuidas de la siguiente manera: municipio de Amapala, 44; municipio de San Francisco de Coray, 34; municipio de Alubarén, 14 y municipio de Reitoca, 64.

Se analizaron 156 muestras, con los primeras 13A y 13B, en búsqueda de ADN de protozoos del género Leishmania. Se detectaron 130 muestras positivas. Una segunda PCR se utilizó para caracterización molecular de Le. infantum con los primer RV1 y RV2. Para esta segunda reacción se analizaron las 130 muestras, incluidas las negativas a Leishmania spp, a manera de confirmación del diagnóstico negativo.

Como resultado para este segundo set de primers, nuevamente las 26 muestras negativas par Leishmania spp con los primers 13A y 13B, mostraron un resultado 
negativo, coincidiendo el resultado con la primera PCR. De los 130 muestras restantes, las 128 mostraron resultados positivos, detectándose en estas muestras ADN del protozoo Leishmania chagasi/ infantum, lo que permitió caracterizar a esta especie como el agente etiológico presente en las lesiones de los pacientes a los cuales pertenecen estas muestras. Las frecuencias absolutas encontradas se observan en el cuadro 2.

Cuadro 2. Distribución de muestras procesadas con cebadores para Leishmania sp y Leishmania infantum/chagasi

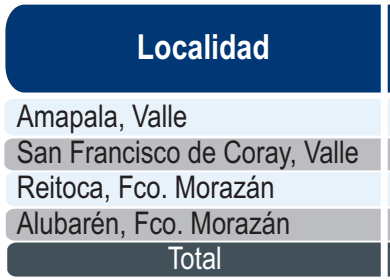

\begin{tabular}{|c|}
\hline $\begin{array}{c}\text { Casos } \\
\text { sospechoso }\end{array}$ \\
\hline 44 \\
34 \\
64 \\
14 \\
\hline 156 \\
\hline
\end{tabular}

\begin{tabular}{|c|}
\hline $\begin{array}{c}\text { Positivos a } \\
\text { Leishmania }\end{array}$ \\
\hline 38 \\
27 \\
60 \\
\hline 5 \\
\hline 130 \\
\hline
\end{tabular}

\begin{tabular}{|c|}
\hline$L$. \\
chagasilinfantum \\
\hline 36 \\
27 \\
60 \\
5 \\
\hline 128 \\
\hline
\end{tabular}

\section{Diversidad de especies del género Lutzomyia}

Por medio de la utilización de criterios (Young y Duncan, 1994) se logró identificar un total de 5,951 Lutzomyias, de la cuales 3,543 (60 \%) son machos y 2,404 (40\%) son hembras con o sin ingesta de sangre. En el cuadro 3 se observa la distribución de insectos capturados por municipio.

Cuadro 3. Distribución de Lutzomyia por municipio

\begin{tabular}{|l|c|c|c|}
\hline \multirow{2}{*}{ Municipio } & \multicolumn{3}{c|}{ Número de Lutzomyias capturadas } \\
\cline { 2 - 4 } & $\boldsymbol{?}$ & $?$ & $?$ \\
\hline Amapala & 772 & 2149 & 2,921 \\
San Francisco de Coray & 731 & 329 & 1,060 \\
\hline Alubarén & 403 & 127 & 5,30 \\
\hline Reitoca & 502 & 938 & 1,951 \\
\hline \multicolumn{1}{|c|}{ Totales } & 2408 & 3543 & 5,951 \\
\hline
\end{tabular}

De esta población de insectos capturados, se identificaron diez especies de Lutzomyias: Lu. cruciata, Lu. chiapanensis, Lu. gomezi, Lutzomyia sp, subgénero Franca, Lu. zeledoni, Lu. sanguinaria, Lu. trapidoi, Lu. longipalpis, Lu. evansi, Lu. cayenensis cayenensis. Siendo Lu. longipalpis, Lu. evansi y Lu. cruciata las de mayor predominio (ver cuadro 4). De los 92 pooles analizados, solamente 1 
pertenece a Lu. longipalpis, que resultó positivo a la detección de parásitos del género Leishmania. Se determinó que Leishmania infantum/chagasi fue la especie de Leishmania encontrada en Lu. longipalpis, encontrándose una tasa de infección del $0.27 \%$.

Cuadro 4. Riqueza específica de cada uno de los municipios estudiados

\begin{tabular}{l|l|c|c|}
\hline \multicolumn{1}{|c|}{ Municipio } & Departamento & $\begin{array}{c}\text { Riqueza } \\
\text { específica }\end{array}$ & \multicolumn{1}{c|}{$\begin{array}{c}\text { Especies de } \\
\text { Lutzomyia }\end{array}$} \\
\hline Amapala & Valle & 8 & $\begin{array}{l}\text { Lu. longipalpis, Lu. cruciata, Lu. chiapanensis, Lu. gomezi, } \\
\text { Lutzomyia sp, subgénero Franca, Lu. zeledoni, Lu. } \\
\text { sanguinaria, Lu. trapidoi }\end{array}$ \\
\hline $\begin{array}{l}\text { San Francisco } \\
\text { de Coray }\end{array}$ & Valle & 4 & $\begin{array}{l}\text { Lu. longipalpis, Lu. chiapanensis, Lu. zeledoni y Lu. } \\
\text { trinidadensis }\end{array}$ \\
\hline Alubarén & $\begin{array}{l}\text { Francisco } \\
\text { Morazán }\end{array}$ & 4 & $\begin{array}{l}\text { Lu. longipalpis, Lu. chiapanensis, Lu. evansi y Lu. } \\
\text { sanguinaria }\end{array}$ \\
\hline Reitoca & $\begin{array}{l}\text { Francisco } \\
\text { Morazán }\end{array}$ & 7 & $\begin{array}{l}\text { Lu. longipalpis, Lu. chiapanensis, Lu. zeledoni, Lu. evansi, } \\
\text { Lu. sanguinaria, Lu. gomezi, Lu. cayenensis cayenensis }\end{array}$ \\
\hline
\end{tabular}

Mediante el empleo del índice de Margalef, se observa (ver cuadro 5) que el municipio con mayor diversidad fue Alubarén $\left(D_{M g}=0.96\right)$, seguido de Amapala $\left(D_{M g}=0.88\right)$, luego se encuentra San Francisco de Coray $\left(D_{M g}=0.43\right)$ y con la diversidad más baja se halla Reitoca $\left(D_{M g}=0.41\right)$. Según lo que nos expone el índice de Margalef, aquellos valores inferiores a 2.0 corresponden a zonas o comunidades con baja diversidad de especies y, por el contrario, aquellos valores superiores a 5.0 indican comunidades con una alta biodiversidad. Teniendo en cuenta la consideración anterior, los cuatro municipios $\left(D_{M g}=1.04\right)$ presentan una baja diversidad de especies de Lutzomyia.

Cuadro 5. Índice de Margalef para los cuatro municipios y para el área de estudio
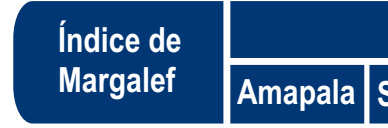

0.88

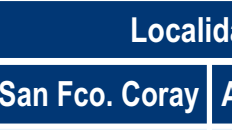

0.43
0.96

\section{Diversidad \\ área de \\ estudio}

1.04

En cuanto a la semejanza en la composición de especies entre localidades medidas a través del coeficiente de Jaccard, estas mostraron una similitud que osciló entre 0.33 y 0.50; es decir, que presentaron una composición de especies de Lutzomyia similar en un $33 \%$ - 50 \% (ver cuadro 6). Esto significa que dichas localidades son independientes en su composición de especies. 
A partir de lo expuesto anteriormente, se puede considerar que los cuatro municipios son diferentes entre sí en cuanto a composición de especies, lo cual las define como comunidades heterogéneas y con una baja diversidad de especies de Lutzomyia dentro de las mismas, según el índice de Margalef.

Cuadro 6. Valores del coeficiente de Jaccard entre las localidades evaluadas

\begin{tabular}{|l|c|c|c|c|}
\hline \multicolumn{1}{|c|}{ Localidad } & Amapala & San Fco. Coray & Alubarén & Reitoca \\
\hline Amapala & --- & --- & --- & -- \\
\hline San Fco. del Coray & 0.33 & --- & --- & --- \\
\hline Alubarén & 0.50 & 0.38 & --- & --- \\
\hline Reitoca & 0.33 & 0.33 & 0.38 & --- \\
\hline
\end{tabular}

Determinación de ingestas alimenticias en especies de Lutzomyia

Se identificaron 5 especies ingurgitadas con sangre, de las cuales a $L u$. zeledoni y $L u$. evansi no fue posible determinarles el origen de la misma. Dentro de las 3 especies a las que sí se les determinó sus ingestas alimenticias: Lu. longipalpis, $L u$. cruciata y Lu. chiapanensis, mostraron preferencia por hábitos alimenticios de Canis familiaris, Gallus gallus y Sus scrofa. No se logró determinar antropofilia en las especies examinadas.

\section{Caracterización macroambiental}

La zona de vida identificada con respecto al sistema Holdrige se corresponde con el bosque seco tropical. Con la información del Atlas Digital de Honduras y la asignación de datos con la extensión geoprocesadora del software ArcView 3.3, se determinó que los suelos en las cuatro regiones son de origen volcánico del cuaternario, de tipo basáltico, con una profundidad media, buen drenaje, $\mathrm{pH}$ de 6.5, de coloración oscura y textura arcilla marga.

De manera local y basada en la clasificación de los suelos por su potencial agrícola, según la Organización de las Naciones Unidas, Amapala presenta suelos asociados a cultivos en general y uso apropiado de bosques, pero medidas de conservación extremas son necesarias dado que presentan tendencia a la corrosión. Según la clasificación de la FAO, los suelos de los 4 municipios son Ustorthents líticos, asociados a partir de rocas ígneas ácidas o metamórficas en topografía montañosa, de clase regosoles. Durante el estudio, se captaron pacientes con LCNU en viviendas ubicadas en alturas mínimas de $-3 \mathrm{msnm}$ y máxima de $732 \mathrm{msnm}$, con un promedio de $291 \mathrm{msnm}$; se registró una temperatura promedio de $37^{\circ} \mathrm{C}$. 


\section{DISCUSIÓN}

En el presente estudio se abordaron aspectos de la ecoepidemiología de la LCNU en cuatro municipios endémicos. Lo novedoso del estudio es que integra herramientas moleculares actuales como el PCR combinado con los SIG.

Históricamente, el municipio de Amapala ha sido considerada una zona endémica a LCNU, sin embargo, desde el año 1991cuando se realizó el trabajo de campo llevado a cabo por Ponce (1991) y la evaluación del banco de muestras realizada por Noyes 1997, no se han conducido estudios para mantener actualizada la información concerniente a la epidemiología de la enfermedad, incluyendo encuestas entomológicas de vectores, ya que solamente existe el registro de casos que acuden a la unidad de salud establecida a nivel municipal.

Dichos registros refieren que para el año 2010 se detectaron 18 casos de LCNU, cifra que aumentó a 22 para el año 2011 (Programa Nacional de Prevención y Control de la Enfermedad de Chagas y Leishmaniasis, Secretaría de Salud de Honduras). En los restantes 3 municipios no existen reportes de las especies de Leishmania y Lutzomyia circulantes en cada una de las zonas.

Durante los 4 meses de muestreo (febrero-mayo) se colectaron 156 muestras de pacientes sospechosos con LCNU, de estos 130 (83.33\%) se lograron caracterizar como Leishmania infantum chagasi, lo que concuerda con datos informados en zonas endémicas de Honduras y Centroamérica (Ponce, 1991; Peraza, 1998; Zeledon, 1993).

En los estudios ecoepidemiológicos de las diferentes formas clínicas de la leishmaniasis, un punto clave corresponde al estudio de las poblaciones de flebotomíneos, teniendo suma relevancia elementos como la identificación taxonómica y el conocimiento de sus hábitos alimenticios, puntos imprescindibles para la incriminación de las especies como vectores biológicos, sobre todo si se logra demostrar el comportamiento antropofílico y zoofílico (Carrillo Robledo, 2010; Kato, 2005).

Otro aspecto de relevancia, y muchas veces difícil de evidenciar, es la infección natural con el agente etiológico cuestionado. El presente trabajo abordó 3 aspectos importantes de la biología de los vectores de la LCNU en Honduras: la actual diversidad de especies de Lutzomyia presentes en la zona de estudio, la determinación de ingestas alimenticias de estas especies identificadas y la detección de infecciones naturales en estos vectores. Se obtuvieron resultados 
relevantes que han permitido actualizar los conocimientos de estas poblaciones de flebótomos en esta zona de estudio. Se identificaron taxonómicamente 10 especies de Lutzomyia presentes en la zonas de estudio, de las cuales Lu. longipalpis fue predominante, seguida por Lu. evansi y Lu. cruciata, lo que concuerda con estudios realizados en Centroamérica y Suramérica (Gonzalez, 2014; Missawa, 2010; Soares, 2010).

De los 92 pooles que se analizaron en búsqueda de parásitos de Leishmania, solo 1 pool de Lu. longipalpis fue en la cual se logró demostrar la infección natural con el protozoo L. infantum/chagasi, resultados que sugieren que esta especie de Lutzomyia es el vector de LCNU en Amapala y concuerda con resultados previos realizados en la en Honduras (Noyes, 1997).

Se identificaron 5 especies ingurgitadas con sangre, de las cuales a Lu. zeledoni y $L u$. evansi no fue posible determinarle el origen de la misma. Dentro de las 3 especies a las que sí se les determinó sus ingestas alimenticias: Lu. longipalpis, Lu. cruciata y Lu. chiapanensis, mostraron preferencia por hábitos alimenticios de Canis familiaris, Gallus gallus y Sus scrofa. No se logró determinar antropofilia en las especies examinadas.

Es importante señalar que estos resultados son consistentes con los hallazgos encontrados en la misma zona de estudio, en donde el perro es el principal reservorio de L.chagasi/infantum en América y en nuestro país (Ponce, 1991; Rondon, 2008; Almeida, 2005); por lo que su papel protagónico en el establecimiento del ciclo domiciliar de la leishmaniasis es indiscutible. Por otra parte, el cerdo (reservorio potencial) y la gallina (refractaria a infecciones por Leishmania sp.), al ser empleados por los flebótomos como fuentes alimenticias importantes, implicaría que la tenencia de estos animales alrededor de las viviendas es un factor de riesgo que favorecería la presencia y mantenimiento de las poblaciones de Lutzomyia en el ambiente peridomiciliar (Sant'anna, 2010; Morrison, 1993).

La descripción macroambiental de las áreas estudiadas refiere que estas zonas presentan características muy propias en donde los vectores se han adaptado a vivir. La caracterización macroambiental también permitió conocer que estas áreas comparten particularidades con otras zonas con reportes previos de LCNU en Nicaragua y Costa Rica (Carrillo Robledo, 2010; Belli, 1993).

En conclusión, el enfoque ecoepidemiológico utilizado en este estudio demostró ser una alternativa para comprender de manera integral la situación de la leishmaniasis cutánea no ulcerada en Honduras. De este modo, se puede obtener información 
valiosa para comprender el comportamiento de la leishmaniasis y así utilizar nuevas herramientas para el diagnóstico, gestión y el control de esta zoonosis.

\section{BIBLIOGRAFÍA}

Almeida, M.A. et al. (2005). Clinical and serological aspects of visceral leishmaniasis in northeast.

Brazilian dogs naturally infected with Leishmania chagasi. Vet Parasitol, 127. 227232.

Bhattacharyya, R. et al. (1996). Development of a genus specific primer set for detection of Leishmania parasites by polymerase chain reaction. FEMS Microbiol Lett, 135. 195-200.

Belli, A. et al. (1993). Epidemiological aspects of the leishmaniasis in Central America. Arch Inst Pasteur Tunis, 70, 489.

Carrillo Robledo, Velez ID. (2010) Manual diagnóstico y control de la leishmaniasis en Centroamérica. Programa de Estudio y Control de Enfermedades Tropicales, Universidad de Antioquia. Colombia.

Gonzalez, C.; Paz, A y Ferro, C. (2014). Predicted altitudinal shifts and reduced spatial distribution of Leishmania infantum vector species under climate change scenarios in Colombia. Acta Trop, 129, 83-90.

Kato, H. et al. (2005). Detection and identification of Leishmania species within naturally infected sand flies in the andean areas of ecuador by a polymerase chain reaction. Am J Trop Med Hyg, 72, 87-93.

Lachaud, L. et al. (2002). Comparison of six PCR methods using peripheral blood for detection of canine visceral leishmaniasis. J Clin Microbiol, 40, 210-215.

Loyola, C. (2007). Phlebotomine fauna (Dyptera: Psychodidae) and species abundance in an endemic area of ACL in southern Minas Gerais, Brazil. Mem Instit Oswaldo Cruz, 202(5), 581-585.

López, M. et al. (1993). Diagnosis of Leishmania using the polymerase chain reaction: a simplified procedure for field work. Am J Trop Med Hyg, 49, 348-356. Moreno, C. (2001). Métodos para medir la biodiversidad. España: M\&T-Manuales. Missawa, N.A. et al. (2010). Lutzomyia longipalpis naturally infected by Leishmania (L.) chagasi in Varzea Grande, Mato Grosso State, Brazil, an area of intense transmission of visceral leishmaniasis. Cad Saude Publica, 26, 2414-2419.

Morrison, A.C.; Ferro, C.; Tesh, R.B. (1993). Host preferences of the sand fly Lutzomyia longipalpis at an endemic focus of American visceral leishmaniasis in Colombia. Am J Trop Med Hyg, 49, 68-75. 